\title{
Tailor-made starch-based conjugates containing well-defined poly(vinyl acetate) and its derivative poly(vinyl alcohol)
}

\author{
D. R. Lu, C. M.Xiao ${ }^{*}$ S. J. Xu, Y. F. Ye \\ College of Material Science and Engineering of Huaqiao University, Quanzhou, 362021, China
}

Received 19 October 2010; accepted in revised form 26 December 2010

\begin{abstract}
Reversible addition-fragmentation chain transfer (RAFT) polymerization was adopted to synthesize starch-based conjugates that possessed controllable architecture and properties. Starch-based xanthate agent was prepared and applied as chain transfer agent to conduct the living/controlled polymerization (LCP) of vinyl acetate, which generated tailor-made conjugates of starch and well-defined poly(vinyl acetate) (SVAc). The relevant derivatives, conjugates of starch and chain length-controlled poly(vinyl alcohol) (SVA), were obtained subsequently. Various characterizations such as Fourier transform infrared spectra (FTIR), ultraviolet-visible spectroscopy (UV), proton nuclear magnetic resonance $\left({ }^{1} \mathrm{H}\right.$ NMR), gel permeation chromatography (GPC), X-ray diffraction (XRD), Thermogravimetric analysis (TGA), and dynamic mechanical thermal analysis (DMTA) were performed to examine the structure of intermediates and the starch-based conjugates. Static contact angle measurements revealed that the hydrophilic character of starch-based conjugates was tunable. Well-defined SVAc was amphiphilic and it was able to self-assemble into size controllable micelles, which was verified by contact angles, transmission electron microscopy (TEM) and dynamic light scattering (DLS) tests. SVA exhibited much higher capability to form physically cross-linked hydrogel than starch did. Both the characteristic of SVAc and SVA were chain length-dependent.
\end{abstract}

Keywords: tailor-made polymers, starch, vinyl acetate, synthesis, properties

\section{Introduction}

Being challenged with environmental and petroleum resources issues, polymers from renewable resources are considered as the future macromolecular materials [1]. Starch is an annually renewable and biodegradable agriculturally derived polysaccharide. It has been receiving growing attention for decades, and is regarded as a promising candidate for developing sustainable materials [2]. Poly(vinyl alcohol) (PVA) is a water soluble synthetic polymer that exhibits several advantages such as biodegradability, biocompatibility and capacity of forming physically cross-linked hydrogel under mild condition [3-4]. Combination of starch and PVA has been mainly documented as the relevant blend [5] and graft copolymer [6].

It is well known that the structure and function of polymers are intimately related. Subtle modulation of chain architecture probably generates new materials with dramatically different properties [7]. Among the tools to define polymers well, reversible addition-fragmentation chain transfer (RAFT) polymerization is a facile, convenient and versatile approach to prepare functionalized polymers with controlled architecture [8-9]. It may be an effective way for combining the advantages of starch and PVA components with controllable macromolecular architectures. As a natural polymer, the structure of

\footnotetext{
${ }^{*}$ Corresponding author, e-mail: congmingxiao@hqu.edu.cn
} (c) BME-PT 
starch is unavailable to be controlled [10]. Thus, one feasible strategy is to attach the precisely tailormade PVA onto starch.

As well known, PVA is easy to be derived from saponification of poly(vinyl acetate) (PVAc). Moreover, Stenzel and her coworkers have revealed that RAFT/MADIX (macromolecular design via interchange of xanthates) polymerization enables the monomer VAc to polymerize in a controlled manner [11]. McLeary group has performed the grafting copolymerization of VAc onto two derivatives of cellulose via RAFT polymerization [12]. But rare attention has been paid to the RAFT polymerization of VAc using abundant starch as substrate [13]. These facts encourage us to conduct the research on conjugating starch with well-defined PVA. This article aims at tailoring the synthesis and properties of starch-based conjugates. Herein, starch-based xanthate-mediated living radical polymerization of VAc and subsequent alcoholysis were carried out to generate two kinds of conjugates. The properties of the conjugates were investigated to find out the characters of the starch-based polymers.

\section{Experimental}

\subsection{Materials}

Water-soluble starch (Shanghai Chemical Agents Ltd. Co., China) was dried before use. Vinyl acetate (VAc, Shanghai Chemical Agents Ltd. Co., China) was purified by distillation. Maleic anhydride (MAn, m.p. $54-55^{\circ} \mathrm{C}$, Shanghai Chemical Agents Ltd. Co., China) and N,N'-Azobisisobutyronitrile (AIBN, Shanghai Chemical Agents Ltd. Co., China) were purified by recrystallizing from benzene (Shanghai Chemical Agents Ltd. Co., China) and anhydrous ethanol (Shanghai Chemical Agents Ltd. Co., China) respectively. Carbon disulfide (Shanghai Chemical Agents Ltd. Co., China) was dried by anhydrous magnesium sulfate (Shanghai Chemical Agents Ltd. Co., China) before used. Dimethyl sulfoxide (DMSO, Shanghai Chemical Agents Ltd. Co., China), anhydrous ethanol, 95\% ethanol (Shanghai Chemical Agents Ltd. Co., China), methanol (Shanghai Chemical Agents Ltd. Co., China), sodium hydroxide (Shanghai Chemical Agents Ltd. Co., China), potassium hydroxide (Shanghai Chemical Agents Ltd. Co., China), pyridine (Shanghai Chemical Agents Ltd. Co., China), N,N'-Dimethyl- formamide (DMF, Shanghai Chemical Agents Ltd. Co., China), potassium ethyl xanthogenate (Shanghai Chemical Agents Ltd. Co., China) and hydrochloric acid $(\mathrm{HCl}$, Shanghai Chemical Agents Ltd. Co., China) were all analytical grade reagents and used as received.

\subsection{Synthesis of starch-based xanthate agent}

Firstly, unsaturated starch-based macromonomer (SM) was prepared according to the literature [14] by the esterification between starch and MAn. Briefly, $24 \mathrm{~g}$ dry starch was dissolved in $28 \mathrm{ml}$ water at $100^{\circ} \mathrm{C}$, mixed with $60 \mathrm{ml} \mathrm{DMF}$ and transferred into a $250 \mathrm{ml}$ flask. A mixture of $24 \mathrm{~g}$ MAn, $24 \mathrm{ml}$ pyridine and $60 \mathrm{ml}$ DMF was added into the flask in droplet under stirring. The reaction mixture was allowed to react for $24 \mathrm{~h}$ with agitation at room temperature. The crude product was precipitated from $95 \%$ ethanol and purified by extracting with $95 \%$ ethanol in a Soxhlet apparatus for $24 \mathrm{~h}$. The dried light yellow powder was pure SM. Yield: $65 \%$. Carbon-carbon double bond percentage: $22.5 \%$ (calculated from titration results). FTIR $\left(\mathrm{KBr}, \mathrm{cm}^{-1}\right)$ : $3450(-\mathrm{OH}), 2931\left(-\mathrm{CH}_{2}-,-\mathrm{CH}_{3}\right), 1732(-\mathrm{C}=\mathrm{O})$, $1638(-\mathrm{C}=\mathrm{C}-)$.

Starch-based xanthate agent (SXA) was prepared according to the literature [15] with some improvements. Briefly, $10 \mathrm{~g} \mathrm{SM}$ was dissolved in $40 \mathrm{ml}$ DMSO at $80^{\circ} \mathrm{C}$. The solution and proper amount of acetic acid were added into a $100 \mathrm{ml}$ round-bottom flask. Constant $\mathrm{HBr}$ gas was bubbled through the solution and the mixture was kept stirring for $24 \mathrm{~h}$ at $60^{\circ} \mathrm{C}$. Then $10 \mathrm{~g}$ potassium ethyl xanthogenate was added and the mixture was allowed to react for another $24 \mathrm{~h}$. After isolating the by-product $\mathrm{KBr}$ by filtration, the crude product was precipitated from $200 \mathrm{ml}$ anhydrous ethanol and purified by $24 \mathrm{~h}-$ extraction with ethanol in a Soxhlet apparatus. The dried dark yellow powder was SXA. Yield: $55 \%$. FTIR $\left(\mathrm{KBr}, \mathrm{cm}^{-1}\right): 3425(-\mathrm{OH}), 2924\left(-\mathrm{CH}_{2}-\right.$, $\left.-\mathrm{CH}_{3}\right), 1042(-\mathrm{C}=\mathrm{S}) . \mathrm{UV}(\mathrm{C}=\mathrm{S}): 385 \mathrm{~nm}\left(\mathrm{n} \rightarrow \pi^{*}\right.$, $\left.\lambda_{\max }\right), 300 \mathrm{~nm}\left(\mathrm{n} \rightarrow \pi^{*}, \lambda\right), \mathrm{H}_{2} \mathrm{O}$ as solvent. ${ }^{1} \mathrm{H}$ NMR $\left(\mathrm{D}_{2} \mathrm{O}, \delta \mathrm{ppm}\right.$ ): 1.12 (a: $\left.\mathrm{O}-\mathrm{CH}_{2}-\mathrm{CH}_{3}, 3 \mathrm{H}, \mathrm{t}\right), 2.59$ (d: $\left.\mathrm{CH}-\mathrm{CH}_{2}-\mathrm{OCO}-, 2 \mathrm{H}, \mathrm{d}\right), 4.06\left(\mathrm{c}:-\mathrm{CH}_{2}-\mathrm{CH}(\mathrm{S})-\mathrm{C}=\mathrm{O}\right.$, 1H, d), 4.43 (e: $\mathrm{O}-\mathrm{CH}_{2}-\mathrm{CH}, 2 \mathrm{H}, \mathrm{d}$, on starch), 4.86 (b: $\mathrm{O}-\mathrm{CH}_{2}-\mathrm{CH}_{3}, 2 \mathrm{H}, \mathrm{q}$ ), 5.01, 5.31 and 3.2-3.7 (f: protons on starch backbone). 


\subsection{SXA-mediated VAc RAFT polymerization}

To prepare conjugates (SVAc) of starch and molecular weight-controlled poly(vinyl acetate), the RAFT polymerization of VAc mediated via starch-based xanthate agent was conducted [11]. Briefly, $0.1 \mathrm{~g}$ SXA was dissolved in $6 \mathrm{ml} \mathrm{DMSO}$ at $80^{\circ} \mathrm{C}$, and cooled down to room temperature, then a mixture of $0.04 \mathrm{~g}$ AIBN and $4 \mathrm{ml}$ VAc was added dropwise. The mixture was transferred into a Schlenk tube which was thoroughly deoxygenated by three consecutive freeze-pump-thaw cycles. Several tubes were kept at $60^{\circ} \mathrm{C}$ water bath and removed at regular time intervals. The reactions were terminated by plunging the tubes into iced water. SVAc was precipitated from plenty of water, filtered, washed with water for three times, and dried. The samples were extracted with anhydrous ethanol for $48 \mathrm{~h}$ to remove the homopolymer. The conversion of monomer was determined by gravimetric method. FTIR ( $\mathrm{KBr}$, $\left.\mathrm{cm}^{-1}\right): 3455$ (-OH), $2931\left(-\mathrm{CH}_{2}-,-\mathrm{CH}-\right), 1754$ $(-\mathrm{C}=\mathrm{O}) . \quad{ }^{1} \mathrm{H} \mathrm{NMR} \quad\left(\mathrm{CDCl}_{3}, \delta \mathrm{ppm}\right): 1.26 \quad$ (a: $\left.\mathrm{O}-\mathrm{CH}_{2}-\mathrm{CH}_{3}, 3 \mathrm{H}, \mathrm{t}\right), 1.64-1.94\left(\mathrm{e}:-\mathrm{CH}_{2}-\mathrm{CH}, 2 \mathrm{H}\right.$, $\mathrm{t}$, in PVAc moiety), 1.96-2.12 (c:-OCO-CH 3 , s, in PVAc moiety), 2.64 (f: $\mathrm{CH}-\mathrm{CH}_{2}-\mathrm{OCO}-, 2 \mathrm{H}, \mathrm{d}$ ), 4.09 (g: $\left.\mathrm{CH}_{2}-\mathrm{CH}-\mathrm{COOH}, 1 \mathrm{H}, \mathrm{t}\right), 4.46(\mathrm{~h}:$ $\mathrm{O}-\mathrm{CH}_{2}-\mathrm{CH}, 2 \mathrm{H}$, d, on starch), 4.72 (b: $\mathrm{O}-\mathrm{CH}_{2}-\mathrm{CH}_{3}$, 2H, q), 4.82-5.05 (d: $-\mathrm{OCO}-\mathrm{CH}-\mathrm{CH}_{2-}, 1 \mathrm{H}, \mathrm{m}$, in PVAc moiety), 5.23, 5.43, and 3.3-3.8 (i: protons on starch backbone).

\subsection{Physical formation of SVAc micelles and SVA hydrogel}

A predetermined amount of SVAc was dissolved in THF to obtain $0.004 \mathrm{~g} / \mathrm{ml}$ solution, and then $1 \mathrm{ml}$ SVAc/THF solution was added into $10 \mathrm{ml}$ water slowly with vigorous stirring and kept at room temperature for $12 \mathrm{~h}$. THF was removed and the SVAc micelles were formed. The final concentration of SVAc micellar solution was $4 \cdot 10^{-4} \mathrm{~g} / \mathrm{ml}$.

The conjugates (SVA) of starch and chain lengthcontrolled PVA were obtained by alcoholysis of SVAc. Briefly, A mixture of $2.0 \mathrm{~g}$ SVAc and $15 \mathrm{ml}$ $5 \mathrm{wt} \% \mathrm{NaOH} /$ methanol was kept refluxing for $2 \mathrm{~h}$. The yellowish-white SVA powder was filtered and dried.

To form physically cross-linked hydrogel, the aqueous solution of $10 \mathrm{wt} \% \mathrm{SVA}$ was subjected to three repeated freezing/thawing cycles, $24 \mathrm{~h}$ at $-16^{\circ} \mathrm{C}$ and $3 \mathrm{~h}$ at ambient temperature.

\subsection{Characterizations}

The powdered SM, SXA, SVAc and SVA were mixed with dry $\mathrm{KBr}$ and compressed into disks respectively. Fourier transform infrared spectra (FTIR) of the samples were recorded using a Nexus 470 FTIR spectrometer (Thermo Fisher Scientific, Waltham, MA, USA). The thio-carbonyl bond $(-\mathrm{C}=\mathrm{S})$ in the structure of SXA was identified with Shimadzu UV-2450 UV-Visible spectrometer (Shimadzu Corporation, Kyoto, Japan). ${ }^{1} \mathrm{H}$ NMR spectra of SXA, SVAc and the hydrolysis product of SVAc were recorded on a Bruker AV400 NMR spectrometer (Bruker BioSpin, Rheinstetten, Germany) using $\mathrm{D}_{2} \mathrm{O}$, DMSO- $\mathrm{d}_{6}$ and $\mathrm{CDCl}_{3}$ as solvent respectively. Thermogravimetric analysis (TGA) of the SM, SVAc and SVA were performed with a TA V2.4F thermoanalyzer (Thermo Fisher Scientific, Waltham, MA, USA). Analyses were conducted over the temperature range from 25 to $800^{\circ} \mathrm{C}$ with a programmed temperature increment of $20^{\circ} \mathrm{C} / \mathrm{min}$ under $\mathrm{N}_{2}$ atmosphere. A mixture of $0.5 \mathrm{~g} \mathrm{SVAc}, 10 \mathrm{ml} 1 \mathrm{M}$ $\mathrm{HCl}$ and $10 \mathrm{ml}$ THF was kept refluxing for 6 days to remove starch component completely. Then the apparent molecular weight and polydispersity index (PDI) of the acidic hydrolysis remainder were measured on a Waters gel permeation chromatography (GPC) with three linear Styragel columns, Waters 1515 pump, and Waters 2414 differential refractive index detector at $30^{\circ} \mathrm{C}$ (Waters Corporation, Milford, MA, USA). THF was used as eluent at a flow rate of $1.0 \mathrm{~mL} / \mathrm{min}$ and polystyrene standards were employed for calibration.

Static contact angle of water on SVAc with defined PVAc, starch, and PVAc films was measured with a JC-2000C contact angle analyzer (Shanghai Zhongchen Digital Technique Equipment Co. Ltd, Shanghai, China), an average of five measurements was taken. The surface morphology of the films was observed with Hitachi S-3500N scanning electron microscope (SEM, Hitachi High-Tech, Tokyo, Japan) at the acceleration voltage of $15 \mathrm{kV}$. For TEM observation [16], about $0.02 \mathrm{ml}$ SVAc micellar solution was deposited on a carbon-coated electron microscopy copper grid. Water was evaporated at ambient temperature and atmospheric pressure. The morphology of the micelles was measured with Hitachi H-7650 TEM (Hitachi High-Tech, Tokyo, Japan) at an acceleration voltage of $80 \mathrm{kV}$. The size of SVAc micelles was measured using a Brookhaven 
Instruments Corporation (Holtsville, NY) ZetaPlus dynamic light scattering (DLS) apparatus at scattering angle of $90^{\circ}$ at $25^{\circ} \mathrm{C}$.

$\mathrm{X}$-ray diffraction (XRD) profiles of the dried SVA hydrogel powder were collected with a Bruker D8Advanced diffractometer (Bruker AXS Inv., Madison WI, USA) using Nickel-filtered $\mathrm{Cu}-\mathrm{K}_{\alpha}$ radiation $(k=0.15406 \mathrm{~nm})$ and scanned from 2 to $60^{\circ}$ at a scan speed of $3 \% \mathrm{~min}$. The viscoelastic properties of the SVA hydrogels were investigated using a dynamic mechanical thermal analysis instrument (DMTA IV, Rhemoteric Scientific Inc., Piscataway, NJ, USA) with compression mode at $25^{\circ} \mathrm{C}$ [17].

\section{Results and discussion}

\subsection{Synthesis of intermediate and controlled polymerization}

Incorporation of PVA will greatly improve the processability, mechanical strength and other properties of starch [18-19], which has been achieved by either radical graft copolymerization or blending means. However, to our best knowledge, no report has mentioned about tuning the architecture and property of starch-based polymers with well-defined PVA so far. RAFT polymerization has the potential to be a key living/controlled polymerization (LCP) method for highly value added fields or large scale applications [20]. Especially it is suitable for synthesizing PVAc, the precursor of PVA, with controlled molecular weight and molecular weight distribution [11]. In view of these, the strategy that performs RAFT polymerization of VAc in the presence of starch-based RAFT agent is adopted to integrate starch and PVA with defined macromolecular architectures.

Two steps are involved in synthesis of starch-based RAFT agents (Figure 1). Macromonomer SM contains $22.5 \% \mathrm{C}=\mathrm{C}$ is derived from the esterification of starch and MAn. By using acetic acid as catalyst, the addition of $\mathrm{HBr}$ to the double bonds on $\mathrm{SM}$ is easily to be carried out. Then the bromium atoms are substituted with xanthogenate groups to obtain SXA. Though two forms of SXA may be formed, both of them contain the xanthogenate functional groups. No matter which one is the major product, either is available to conduct the expected starchbased xanthate-mediated living radical polymerization of VAc. According to the Markovnikov addition principle, the probable major intermediates and the target product are 3, 5 and 7.

The structures of SM and SXA are confirmed with FTIR (Figure 2), UV and ${ }^{1} \mathrm{H}$ NMR (Figure 3a). The characteristic absorption bands on the FTIR spectra of $\mathrm{SM}$ at 1732 and $1638 \mathrm{~cm}^{-1}$ are attributed to the stretching vibration of $-\mathrm{C}=\mathrm{O}$ and $\mathrm{C}=\mathrm{C}$. An absorption peak appears at $1042 \mathrm{~cm}^{-1}$ on the FTIR spectra and UV absorptions of thio-carbonyl bond $(-\mathrm{C}=\mathrm{S})$ exhibit at 300 and $38 \mathrm{~nm}$, which verify the samples contain $-\mathrm{C}=\mathrm{S}$ groups. In addition, the ${ }^{1} \mathrm{H}$ NMR analysis results also demonstrate SXA has been synthesized successfully. The proton signals at $4.43,5.01,5.31$ and $3.2 \sim 3.7 \mathrm{ppm}$ belong to the

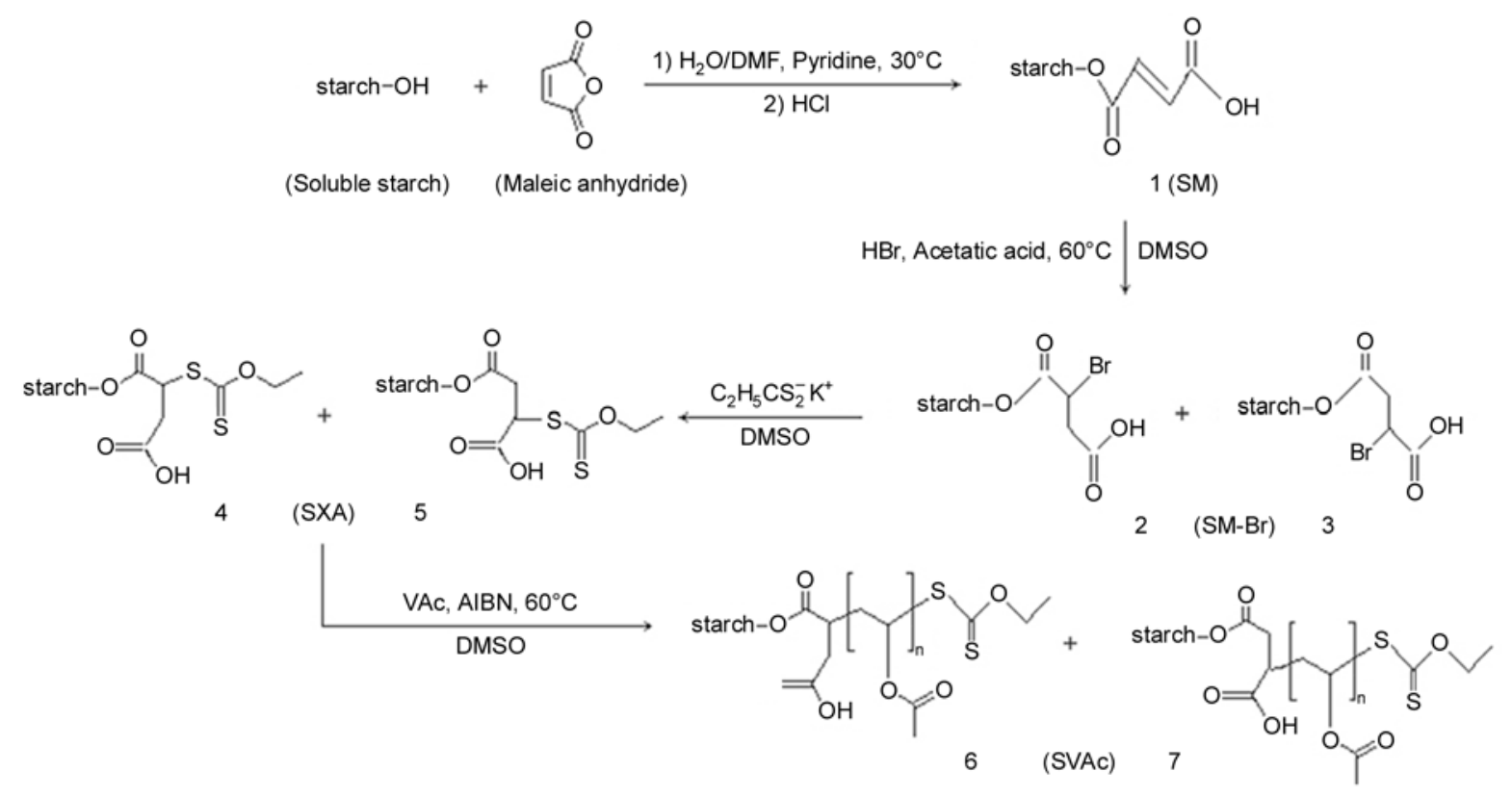

Figure 1. Route of controlled incorporation of well-defined PVAc onto starch via RAFT polymerization 


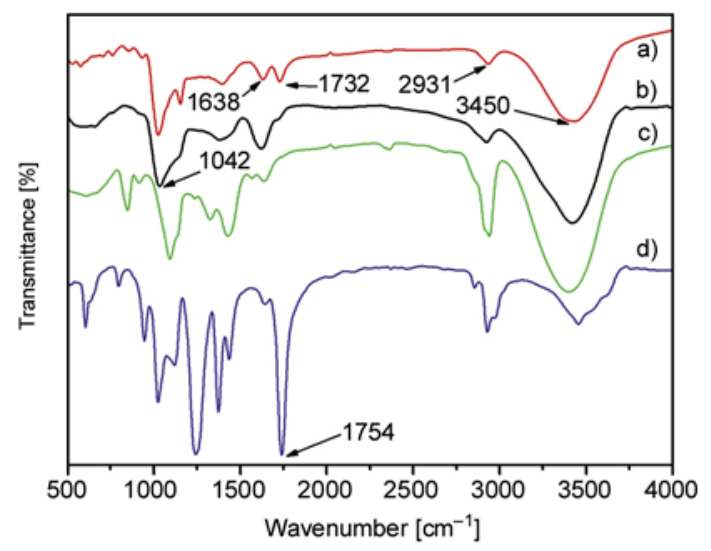

Figure 2. FTIR spectra of SM (a), SXA (b), SVA (c) and SVAc (d)

starch backbone. And the peaks at 1.12, 4.06 and $4.86 \mathrm{ppm}$ indicate that xanthogenate group has been bonded onto starch backbone.

Owing to the characteristic structure of VAc monomer, few RAFT agents are available for its controlled polymerization [21]. Fortunately, polysaccharides can be played as a leaving group to mediate the RAFT polymerization [12, 22-23]. The utilization of polysaccharides-based RAFT agent not only retains the characteristics of natural polymers, but also forms well-defined structure. In current study, SXA is used as macro-RAFT agent and the controlled polymerization of VAc is carried out as expected. The enhanced absorption peak at $1754 \mathrm{~cm}^{-1}$ on the FTIR spectra of corresponding polymer (Figure 2) is attributed to carbonyl group, which suggests that the sample is the target product SVAc. On the FTIR spectra of the alcoholysis derivatives of SVAc (Figure 2), the characteristic absorption band of carbonyl group has disappeared whereas the absorption band of hydroxyl group is strengthened. This proof indicates that SVAc has converted into SVA completely. ${ }^{1} \mathrm{H}$ NMR spectra (Figure 3b) also verify the structure of SVAc. The signs at 1.64 1.94, 1.96 2.12 and 4.82 5.05 ppm are related to the protons on PVAc chain. The peaks at 2.64, 4.09, 4.46, 3.3-3.8, 5.23 and 5.43 ppm belong to that on the starch segment. And the protons of xanthogenate group show their chemical shifts at 1.26 and $4.72 \mathrm{ppm}$.

Figure 4 exhibits the thermal degradation profiles of SM, SVAc and SVA. Their thermal stabilities also reveal the success of the polymerization and alcoholysis reaction. The initial difference of weight loss among SM, SVAc and SVA reflects their water content or hydrophilicity variance. It is found that both SVAc and SVA show two degradation platforms. The first stages $\left(200-350^{\circ} \mathrm{C}\right)$ are assigned to both the decomposition of SM backbone and PVAc segments, and the second ones $\left(390-480^{\circ} \mathrm{C}\right)$ are related to the PVAc or PVA moiety [24]. Since both SM and SVA contain more hydroxyl groups than SVAc, and there are carboxyl groups on the backbone of SM, the ability to form intermolecular hydrogen-bond is in the following order: SM $>$ SVA $>$ SVAc. As a result, the final residual weight fraction of the samples is in the same order. These phenomena confirm the structures of SM, SVAc and SVA once more.
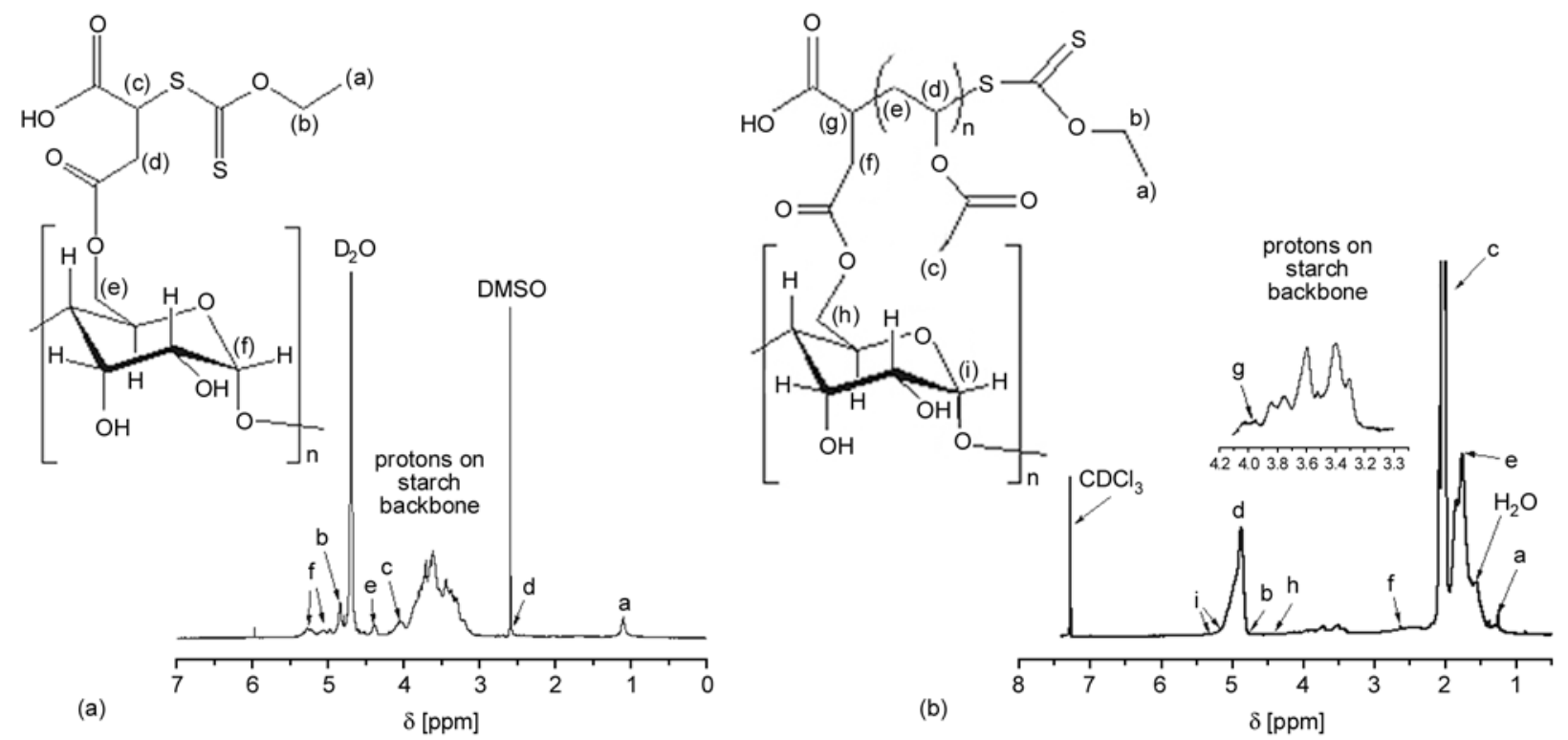

Figure 3. ${ }^{1} \mathrm{H}$ NMR spectra of Starch-based xanthate agent (a) and SVAc (b) 


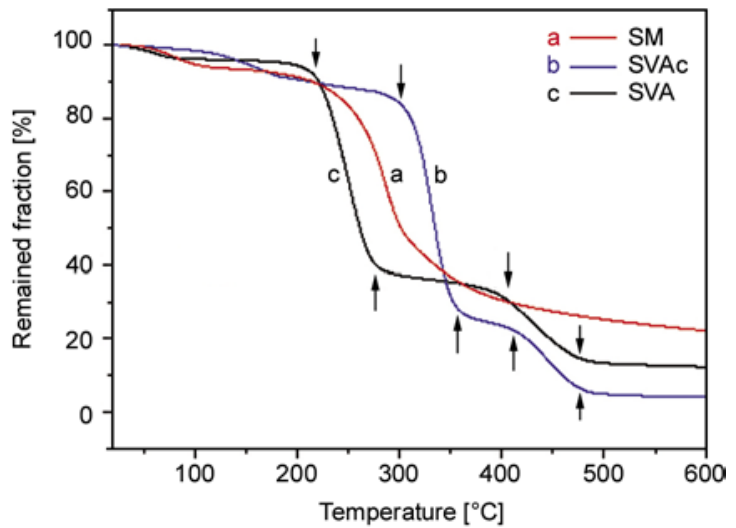

Figure 4. Thermogravimetric analysis profiles of SM (a), SVAc $\left(\mathrm{b}, M_{\mathrm{n}}=8.6 \cdot 10^{4}\right)$ and its derivative SVA (c)

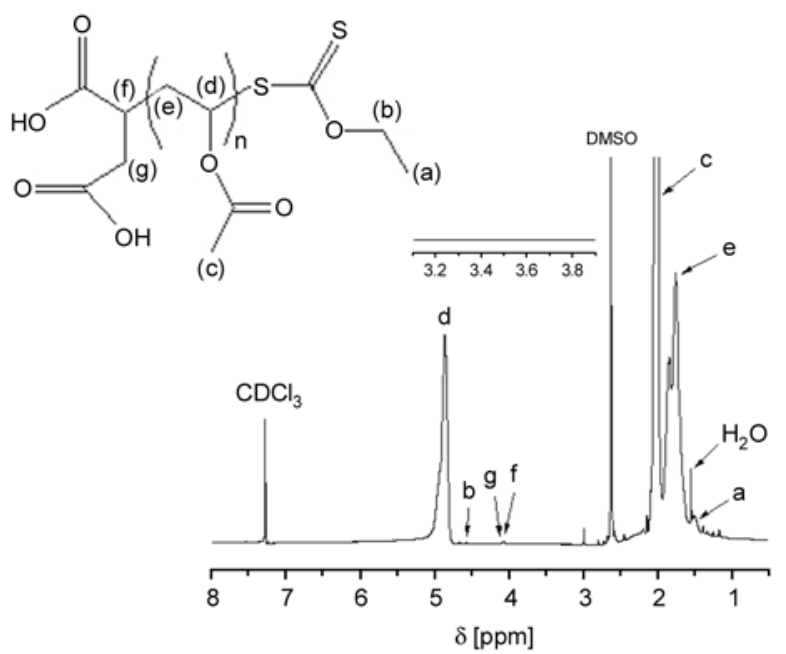

Figure 5. ${ }^{1} \mathrm{H}$ NMR spectrum of the hydrolysis remainder of SVAc

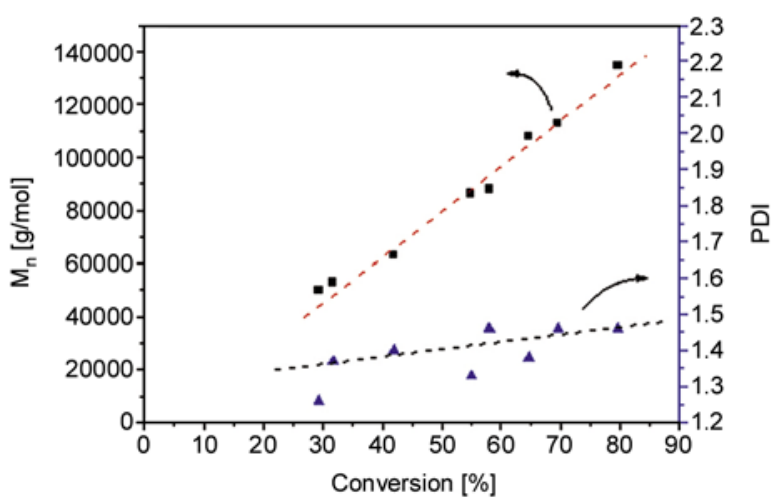

Figure 6. Number-average molecular weight $\left(M_{\mathrm{n}}\right)$ and the polydispersity index (PDI) as the functions of monomer conversion for vinyl acetate RAFT polymerization mediated via starch-based xanthate agent, conditions: $2.44 \cdot 10^{-4} \mathrm{~mol} / 1 \mathrm{AIBN}$, $4.33 \cdot 10^{-2} \mathrm{~mol} / 1 \mathrm{VAc}, 0.1 \mathrm{~g}$ starch-based xanthate agent, $6 \mathrm{ml} \mathrm{DMSO}, 60^{\circ} \mathrm{C}$

To verify starch component of SVAc is removed thoroughly, the structure of acidolysis residue is

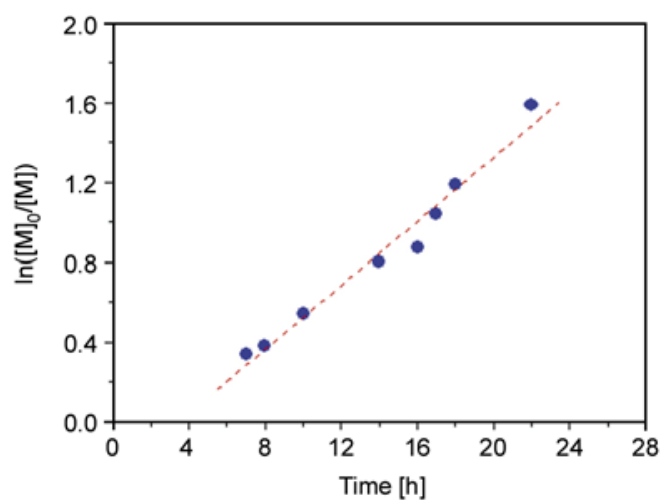

Figure 7. Kinetic curve of RAFT polymerization of vinyl acetate mediated via starch-based xanthate agent $\left(2.44 \cdot 10^{-4} \mathrm{~mol} / \mathrm{l}\right.$ AIBN, $4.33 \cdot 10^{-2} \mathrm{~mol} / 1 \mathrm{VAc}$, $0.1 \mathrm{~g}$ starch-based xanthate agent, $6 \mathrm{ml} \mathrm{DMSO}$, at $\left.60^{\circ} \mathrm{C}\right)$

characterized with the ${ }^{1} \mathrm{H}$ NMR spectra (Figure 5). The signals at 3.2-3.7 ppm have disappeared, which suggests starch is removed completely and the structure of PVAc is kept during the acidolysis. GPC analysis results of the hydrolysis products reveal the dependence of the number-average molecular weight $\left(M_{\mathrm{n}}\right)$ and PDI on monomer conversions (Figure 6). Mn values increase almost linearly with monomer conversions. PDI ranges from 1.26 to 1.46 , which is rather low. The rate plot, $\ln \left([M]_{0} /[M]\right)$ versus time, is almost linear (Figure 7), which indicates the propagating radical concentration is invariant during the polymerization. These results are consistent with the characteristics of LCP [25-26]. In other words, the reaction has implemented as designed.

\subsection{Tunable micellization of SVAc and gelation of SVA}

SVAc is composed of hydrophilic starch and hydrophobic PVAc segments, which indicates that SVAc may be amphiphilic. To evaluate the hydrophilic character of SVAc, the films of starch, PVAc and SVAc are prepared by spin coating. The surface of the films is smooth, as observed with scanning electron microscope (data not shown), which suggests the effect of the surface roughness on the contact angle value can be neglected. Thus, the contact angle values of the samples only rely on their structure. The static contact angle values of water on the starch, PVAc and SVAc indicate that the hydrophobicity of SVAc is intervenient between starch and PVAc (Figure 8). Furthermore, the hydrophobicity of the 


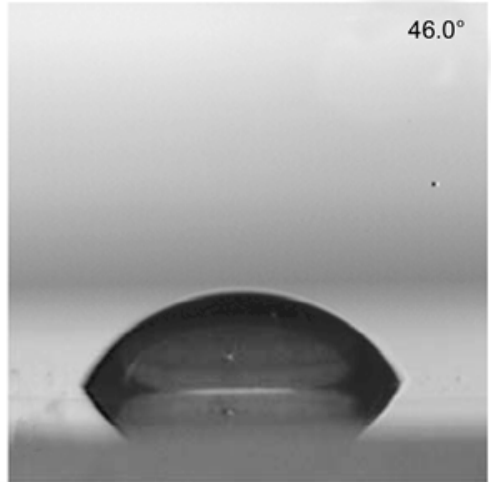

a)

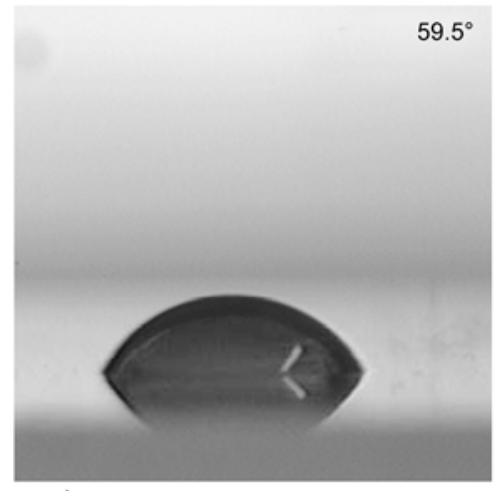

c)

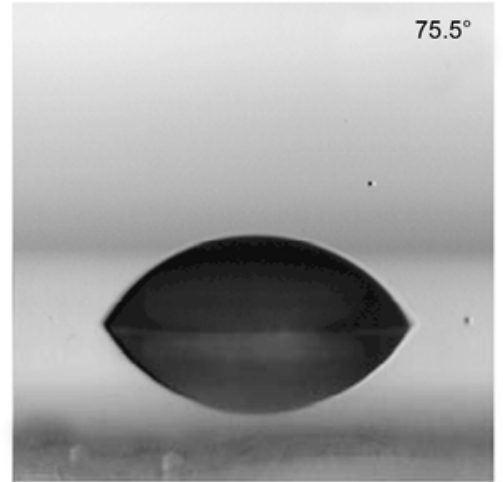

b)

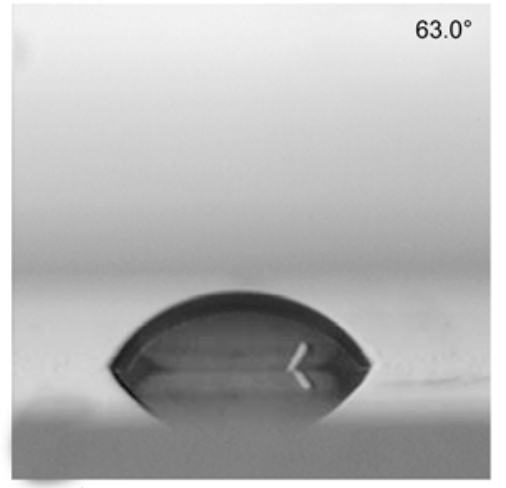

d)

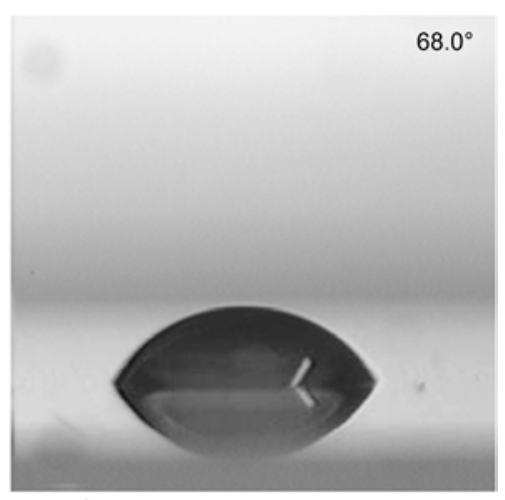

Figure 8. Contact angle of water on (a) starch, (b) PVAc $\left(M_{\mathrm{n}}=1.7 \cdot 10^{5}\right)$, (c) SVAc-I $\left(M_{\mathrm{n}}=5.3 \cdot 10^{4}\right.$, (d) SVAc-II $\left(M_{\mathrm{n}}=\right.$ $\left.8.6 \cdot 10^{4}\right)$ and (e) SVAc-III $\left(M_{\mathrm{n}}=1.3 \cdot 10^{5}\right)$

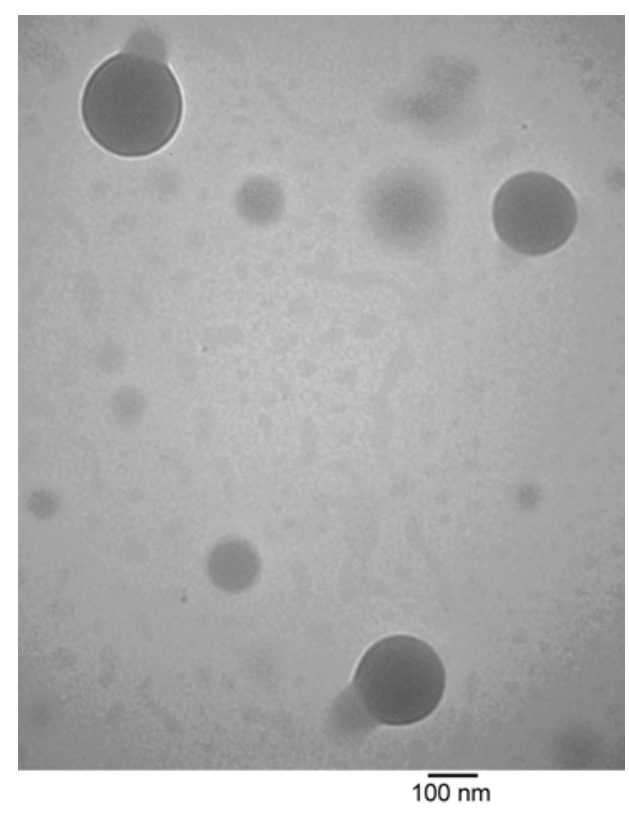

Figure 9. TEM image of SVAc micelle (the solution concentration is $4 \cdot 10^{-4} \mathrm{~g} / \mathrm{ml}$ ), and the size distributions of SVAc micelles assembled in aqueous solution: (1) SVAc-I, (2) SVAc-II, (3) SVAc-III

SVAc increases with the chain length of hydrophobic PVAc segment. It can be inferred that the difference in solubility of two segments in water may result in forming core-corona type micelles [27]. As
Table 1. Hydrophobicity and micellar mean size of SVAc with different molecular weight and its distribution

\begin{tabular}{|l|r|c|c|c|}
\hline Sample & $\begin{array}{c}\mathbf{M}_{\mathbf{n}}^{\mathbf{a}, \mathbf{b}} \\
{[\mathbf{g} / \mathbf{m o l}]}\end{array}$ & PDI $^{\mathbf{b}}$ & $\begin{array}{c}\text { Contact angle } \\
{\left[{ }^{\circ} \mathbf{l}\right.}\end{array}$ & $\begin{array}{c}\text { Mean diameter } \\
{[\mathbf{n m}]}\end{array}$ \\
\hline SVAc-I & 53033 & 1.37 & 59.5 & 137.5 \\
\hline SVAc-II & 86383 & 1.33 & 63.0 & 153.0 \\
\hline SVAc-III & 134659 & 1.46 & 68.0 & 186.1 \\
\hline
\end{tabular}

${ }^{\mathrm{a}} M_{\mathrm{n}}$ is the molecular weight of PVAc, the hydrolysis product of SVAc

${ }^{\mathrm{b}} M_{\mathrm{n}}$ and PDI are determined with GPC

shown in Figure 9, the morphology of SVAc micelles is regular sphere and disperses well without aggregation. The diameter of the micelles is around 100 $200 \mathrm{~nm}$. In addition, DLS measurement results narrate that the size of the micelles in aqueous solution increases with the chain length of PVAc segment (Table 1). Evidently, the hydrophobicity and selfassembly behavior of SVAc depend on molecular weight and are controllable.

It is anticipated that the incorporation of PVA segment will improve the properties of starch evidently. SVAc can be readily converted into SVA by the classical saponification reaction [28]. Herein, what we concerned is the capacity of SVA forming hydrogel in mild physical way, as this property is 


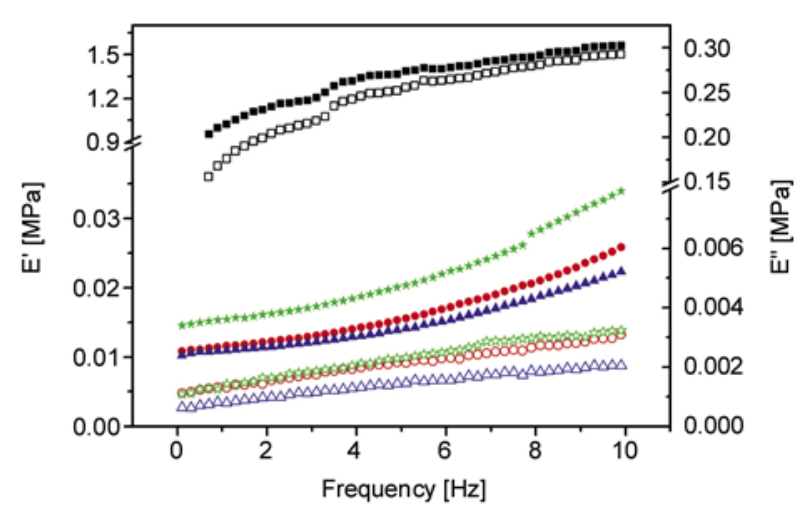

Figure 10. Effect of chain length on the storage modulus and loss modulus of starch and SVA. Storage modulus ( $E^{\prime}$, full symbols), loss modulus ( $E^{\prime \prime}$, open symbols); starch ( $\square$ and $\square)$; SVA-I ( $\Delta$ and $\Delta)$; SVA-II $(\bullet$ and $\circ)$, and SVA-III $(\star$ and $\downarrow)$

quite useful for medical applications such as drug delivery and tissue engineering [29]. To compare the relevant information, DMTA measurements of starch and SVA gels with controlled chain length are implemented. Unlike SVA, starch is difficult to form physically cross-linked hydrogel via freezing/ thawing cycles [6]. Noting a starch gel obtained from $16 \sim 33 \%$ aqueous solution and three freezing/ thawing cycles will disaggregate into powder after being kept in the water for twelve hours, the starch gel used for DMTA test in this article is prepared by subjecting a $50 \%$ aqueous solution of starch to three freezing/thawing cycles. Figure 10 shows the storage modulus $\left(E^{\prime}\right)$ and loss modulus $\left(E^{\prime \prime}\right)$ of starch gel and SVA hydrogels. $E^{\prime}$ and $E^{\prime \prime}$ of starch gel increase with the frequency and level off at last. $E^{\prime}$ of starch gel is about five times higher than its $E^{\prime \prime}$. DMTA analysis results of SVA hydrogels reflect that their mechanical properties are sensitive to the PVA network structure [30]. In the range of $0.1 \sim 10 \mathrm{~Hz}$, the relationships between $E^{\prime}$ (or $E^{\prime \prime}$ ) and frequency of SVA hydrogels are linear. $E^{\prime}$ of SVA hydrogels are ten times greater than their $E^{\prime \prime}$ in value. Moreover, $E^{\prime}$ and $E^{\prime \prime}$ of SVA hydrogels increase as the chain length of PVA segments increases. The crystallinity of the dried SVA hydrogels calculated from the XRD profiles [31] are $29.4,33.6$ and $37.1 \%$ for SVA-1, SVA-3 and SVA-5 respectively. This indicates the relationship between the physical crosslink degree of SVA hydrogel and the molecular weight of PVA segment is in direct ratio and interprets the effect of $M_{\mathrm{n}}$ on $E^{\prime}$ well. These results demonstrate the formation of SVA hydrogels [32]. Noting the concentration of SVA aqueous solution for forming hydrogel is just $10 \%$, it is evident that the incorporation of PVA segment greatly enhance the capability of starch to form physical 3D network structure. Moreover, such ability can be tuned with the molecular weight of PVA.

\section{Conclusions}

Developing starch-based polymers may offer an effective route to enhance the added-value of natural biopolymers and provide an answer for environmental issue. Integration of starch with synthetic PVA is one of the possible ways to this target. Tailoring the chain length of PVA may offer an approach to modulate the properties of starch-PVA biohybrid. In view of these, the conjugate of starch and welldefined PVAc, is developed by conducting starchbased xanthate agent mediated-RAFT polymerization of vinyl acetate. Another starch-based conjugate, SVA, is obtained from its precursor SVAc subsequently. Two conjugates show some interesting properties: SVAc self-assembling into spheral micelles and SVA forming physically cross-linked hydrogel. Moreover, the characteristics of SVAc and SVA are linear-dependence on the molecular weight of PVAc or PVA segments, and the chain lengths of PVAc or PVA segments are controllable. As starch and PVA are biocompatible, SVA may be a potential candidate for biomedical applications. The presented method may also provide an available way for tailor-making starch-based functional materials.

\section{Acknowledgements}

This work was supported by the Natural Science Foundation of Fujian Province of China (No. 2010J01291 or E1010026). The authors were grateful to Mr. S. Liu, H. Z. Zhang and C. H. Yuan for their help to do DLS, NMR and GPC measurements.

\section{References}

[1] Gandini A.: Polymers from renewable resources: A challenge for the future of macromolecular materials. Macromolecules, 41, 9491-9504 (2008). DOI: $10.1021 / \mathrm{ma} 801735 \mathrm{u}$

[2] Lu D. R., Xiao C. M., Xu S. J.: Starch-based completely biodegradable polymer materials. Express Polymer Letters, 3, 366-375 (2009).

DOI: 10.3144 /expresspolymlett.2009.46 
[3] Chiellini E., Corti A., D’Antone S., Solaro R.: Biodegradation of poly (vinyl alcohol) based materials. Progress in Polymer Science, 28, 963-1014 (2003). DOI: 10.1016/S0079-6700(02)00149-1

[4] Ricciardi R., D’Errico G., Auriemma F., Ducouret G., Tedeschi A. M., De Rosa C., Lauprêtre F., Lafuma F.: Short time dynamics of solvent molecules and supramolecular organization of poly (vinyl alcohol) hydrogels obtained by freeze/thaw techniques. Macromolecules, 38, 6629-6639 (2005).

DOI: $10.1021 / \mathrm{ma} 0506031$

[5] Spiridon I., Popescu M. C., Bodârălu R., Vasile C.: Enzymatic degradation of some nanocomposites of poly(vinyl alcohol) with starch. Polymer Degradation and Stability, 93, 1884-1890 (2008).

DOI: $10.1016 /$ j.polymdegradstab.2008.07.017

[6] Xiao C. M., Yang M. L.: Controlled preparation of physical cross-linked starch-g-PVA hydrogel. Carbohydrate Polymers, 64, 37-40 (2006).

DOI: $10.1016 /$ j.carbpol.2005.10.020

[7] Sumerlin B. S., Vogt A. P.: Macromolecular engineering through click chemistry and other efficient transformations. Macromolecules, 43, 1-13 (2010). DOI: $10.1021 / \mathrm{ma} 901447 \mathrm{e}$

[8] Xu J. T., Tao L., Boyer C., Lowe A. B., Davis T. P.: Combining thio-bromo 'click' chemistry and RAFT polymerization: A powerful tool for preparing functionalized multiblock and hyperbranched polymers. Macromolecules, 43, 20-24 (2010).

DOI: $10.1021 / \mathrm{ma} 902154 \mathrm{~h}$

[9] Boyer C., Bulmus V., Davis T. P., Ladmiral V., Liu J. Q., Perrier S.: Bioapplications of RAFT polymerization. Chemical Reviews, 109, 5402-5436 (2009).

DOI: $10.1021 / \mathrm{cr} 9001403$

[10] Angelova N., Hunkeler D.: Rationalizing the design of polymeric biomaterials. Trends in Biotechnology, 17, 409-421 (1999).

DOI: 10.1016/S0167-7799(99)01356-6

[11] Bernard J., Favier A., Zhang L., Nilasaroya A., Davis T. P., Barner-Kowollik C., Stenzel M. H.: Poly(vinyl ester) star polymers via xanthate-mediated living radical polymerization: From poly(vinyl alcohol) to glycopolymer stars. Macromolecules, 38, 5475-5484 (2005).

DOI: $10.1021 / \mathrm{ma} 050050 \mathrm{u}$

[12] Fleet R., McLeary J. B., Grumel V., Weber W. G., Matahwa H., Sanderson R. D.: RAFT mediated polysaccharide copolymers. European Polymer Journal, 44, 2899-2911 (2008).

DOI: 10.1016/j.eurpolymj.2008.06.042

[13] Tizzotti M., Charlot A., Fleury E., Stenzel M., Bernard J.: Modification of polysaccharides through controlled/living radical polymerization grafting - Towards the generation of high performance hybrids. Macromolecular Rapid Communications, 31, 1751-1772 (2010).

DOI: $10.1002 /$ marc. 201000072
[14] Xiao C. M., Ye J.: Preparation of the carboxylic derivates of starch with maleic anhydride (in Chinese). Chinese Journal of Applied Chemistry, 22, 643-646 (2005).

[15] Fleet R., McLeary J. B., Grumel V., Weber W. G., Matahwa H., Sanderson R. D.: Preparation of new multiarmed RAFT agents for the mediation of vinyl acetate polymerization. Macromolecular Symposium, 255, 8-19 (2007).

DOI: $10.1002 /$ masy.200750902

[16] Li Y. G., Zhang Y. Q., Yang D., Feng C., Zhai S. J., Hu J. H., Lu G. L., Huang X. Y.: Well-defined amphiphilic graft copolymer consisting of hydrophilic poly(acrylic acid) backbone and hydrophobic poly(vinyl acetate) side chains. Journal Polymer Science Part A: Polymer Chemistry, 47, 6032-6043 (2009).

DOI: $10.1002 /$ pola.23646

[17] Pan Y. S., Xiong D. S., Gao F.: Viscoelastic behavior of nano-hydroxyapatite reinforced poly(vinyl alcohol) gel biocomposites as an articular cartilage. Journal of Materials Science: Materials in Medicine, 19, 19631969 (2008). DOI: $10.1007 / \mathrm{s} 10856-007-3280-6$

[18] Shi R., Zhu A., Chen D. F., Jiang X. J., Xu X. C., Zhang L. Q., Tian W.: In vitro degradation of starch/ PVA Films and biocompatibility evaluation. Journal of Applied Polymer Science, 115, 346-357 (2010). DOI: 10.1002/app.31136

[19] Samaha S. H., Nasr H. E., Hebeish A.: Synthesis and characterization of starch-poly(vinyl acetate) graft copolymers and their saponified form. Journal of Polymer Research, 12, 343-353 (2005). DOI: $10.1007 / \mathrm{s} 10965-004-7937-2$

[20] Barner-Kowollik C., Perrier S.: The future of reversible addition fragmentation chain transfer polymerization. Journal of Polymer Science Part A: Polymer Chemistry, 46, 5715-5723 (2008).

DOI: $10.1002 /$ pola. 22866

[21] Coote M. L., Radom L.: Substituent effects in xanthate-mediated polymerization of vinyl acetate: $\mathrm{Ab}$ initio evidence for an alternative fragmentation pathway. Macromolecules, 37, 590-596 (2004).

DOI: $10.1021 / \mathrm{ma} 035477 \mathrm{k}$

[22] Barsbay M., Güven O., Stenzel M. H., Davis T. P., Barner-Kowollik C., Barner L.: Verification of controlled grafting of styrene from cellulose via radiationinduced RAFT polymerization. Macromolecules, 40, 7140-7147 (2007).

DOI: $10.1021 / \mathrm{ma} 070825 \mathrm{u}$

[23] Roy D., Knapp J. S., Guthrie J. T., Perrier S.: Antibacterial cellulose fiber via RAFT surface graft polymerization. Biomacromolecules, 9, 91-99 (2008). DOI: $10.1021 / \mathrm{bm} 700849 \mathrm{j}$ 
[24] Rimez B., Rahier H., Van Assche G., Artoos T., Biesemans M., Van Mele B.: The thermal degradation of poly(vinyl acetate) and poly(ethylene-co-vinyl acetate), Part I: Experimental study of the degradation mechanism. Polymer Degradation and Stability, 93, 800-810 (2008).

DOI: $10.1016 /$ j.polymdegradstab.2008.01.010

[25] Perrier S., Davis T. P., Carmichael A. J., Haddleton D. M.: First report of reversible addition-fragmentation chain transfer (RAFT) polymerisation in room temperature ionic liquids. Chemical Communications, 19, 2226-2227 (2002).

DOI: $10.1039 / \mathrm{b} 206534 \mathrm{~g}$

[26] Köllisch H. S., Barner-Kowollik C., Ritter H.: Amphiphilic block copolymers based on cyclodextrinhost-guest complexes via RAFT-polymerization in aqueous solution. Chemical Communications, 9, $1097-$ 1099 (2009).

DOI: $10.139 / \mathrm{b} 818897 \mathrm{a}$

[27] Podríguez-Hernández J., Chécot F., Gnanou Y., Lecommandoux S.: Toward 'smart' nano-objects by self-assembly of block copolymers in solution. Progress in Polymer Science, 30, 691-724 (2005).

DOI: $10.1016 /$ j.progpolymsci.2005.04.002
[28] Fanta G. F., Burr R. C., Doane W. M., Russell C. R.: Graft polymerization of vinyl acetate onto starch. Saponification to starch-g-poly(vinyl alcohol). Journal of Applied Polymer Science, 23, 229-240 (1979). DOI: 10.1002/app.1979.070230121

[29] Hoffman A. S.: Hydrogels for biomedical applications. Advanced Drug Delivery Reviews, 43, 3-12 (2002). DOI: 10.1016/S0169-409X(01)00239-3

[30] Cascone M. G.: Dynamic-mechanical properties of bioartificial polymeric materials. Polymer International, 43, 55-69 (1997).

DOI: 10.1002/(SICI)1097-0126(199705)43:1<55::AIDPI762>3.0.CO;2-\#

[31] Xiao C. M., Geng N. N.: Tailored preparation of dual phase concomitant methylcellulose/poly(vinyl alcohol) physical hydrogel with tunable thermosensivity. European Polymer Journal, 45, 1086-1091 (2009). DOI: $10.1016 /$ j.eurpolymj.2009.01.011

[32] Reinicke S., Schmelz J., Lapp A., Karg M., Hellweg T., Schmalz H.: Smart hydrogels based on double responsive triblock terpolymer. Soft Matter, 5, 2648-2657 (2009).

DOI: $\underline{10.139 / \mathrm{b} 900539 \mathrm{k}}$ 